\title{
ELEMENTARY PROOF OF THE RUDIN-CARLESON AND THE F. AND M. RIESZ THEOREMS
}

\author{
RAOUF DOSS
}

\begin{abstract}
A very elementary proof is given of the theorem that on a set of measure zero on $T$, any continuous function is equal to a continuous function of analytic type. The same elementary method proves that a measure of analytic type is absolutely continuous.
\end{abstract}

A complex Borel measure $\mu$ on $T$, in particular an $f \in L^{1}(T)$, is said to be of analytic type if

$$
a_{n}=(2 \pi)^{-1} \int_{T} e^{-i n t} d \mu(t)=0, \quad n=-1,-2, \ldots
$$

The theorems mentioned in the title are:

Rudin-CaRleson Theorem. Let $F$ be a closed subset of $T$ of Lebesgue measure zero. If $\varphi$ is a continuous function on $F$, then there is a continuous function $f$, of analytic type, such that

$$
\begin{gathered}
f(t)=\varphi(t), \quad t \in F, \\
\sup _{t \in T}|f(t)| \leqslant M \sup _{t \in F}|\varphi(t)|
\end{gathered}
$$

where $M$ is a constant. (Rudin proves that $M=1$. See [8] and [1].)

The First F. AND M. RIESz TheOREM. If the function $f$ in $L^{1}(T)$ is of analytic type and if $f$ vanishes on a set $S^{*}$ of positive measure, then $f=0$.

The Second F. AND M. Riesz Theorem. If a complex Borel measure $\mu$ on $T$ is of analytic type, then $\mu$ is absolutely continuous (with respect to Lebesgue measure). See [7].

The proofs of these theorems most often use boundary values of functions analytic in the unit disc and the theory of $H^{p}$-spaces. For the Second F. and $M$. Riesz Theorem, for example, see three variants in [3], [5] and [9]; other proofs of that theorem use Hilbert-space theory: see e.g. [2] and [4]; a direct short proof is given in [6].

The aim of the present paper is to present a method which gives an elementary proof of all the above theorems.

Received by the editors August 7, 1980; presented to the "Séminaire d'Analyse Harmonique d'Orsay", June 13, 1980.

1980 Mathematics Subject Classification. Primary 42A68, 42A72; Secondary 26A15. 
LEMMA. Let $F$ be a closed subset of $T$ of measure zero and $\varphi$ a continuous function on $F$. Given $\varepsilon>0$ and an open set $G \supset F$ there is a continuous function $g$ of analytic type such that

$$
\begin{gathered}
\sup _{t \in F}|g(t)-\varphi(t)|<\varepsilon \sup _{t \in F}|\varphi(t)|, \\
|g(t)|<\varepsilon, \quad t \notin G, \\
\sup _{t \in T}|g(t)|<3 \sup _{t \in F}|\varphi(t)| .
\end{gathered}
$$

Proof. Without loss of generality we may assume that $\sup _{t \in F}|\varphi(t)|=1$ and also that $\varphi$ is a trigonometric polynomial

$$
\varphi(t)=\sum_{|k|<m} \alpha_{k} e^{i k t}
$$

such that

$$
|\varphi(t)|<\varepsilon / 3, \quad t \notin G .
$$

Let $e^{-A}=\varepsilon$ and let $h$ be a continuous function on $T$, lying between $-2 A$ and $2 \varepsilon$, such that

$$
|h(t)+2 A|<\varepsilon, \quad t \in F .
$$

Since $m(F)=0$ we may take $\|h\|_{1}$ arbitrarily small and hence we may suppose $\hat{h}(k)=0,|k|<m$. Take a Fejér sum $p$ of $h$ such that $|p(t)+2 A|<\varepsilon, t \in F$. We write

$$
p(t)=\sum_{k<-m} \beta_{k} e^{i k t}+\sum_{k>m} \beta_{k} e^{i k t}=p^{-}(t)+p^{+}(t)
$$

where

$$
p^{+}(t)=\sum_{k>m} \beta_{k} e^{i k t}
$$

We have $\operatorname{Re}\left(p^{+}\right)=p / 2<\varepsilon$. Put now

$$
g(t)=\varphi(t)\left[1-e^{p^{+}(t)}\right]
$$

The expansion of [1- $\left.e^{p^{+}(t)}\right]$ is of the form $\Sigma_{k>m} \gamma_{k} e^{i k t}$. The function $g$ is therefore continuous of analytic type. We have

$$
|g(t)-\varphi(t)|=|\varphi(t)|\left|e^{p^{+}(t)}\right|<e^{p / 2}<e^{-A+\varepsilon}<2 \varepsilon \quad(t \in F) .
$$

Moreover

$$
\begin{aligned}
& |g(t)|<|\varphi(t)|\left|1-e^{p^{+}(t)}\right|<1+e^{e}<3 \quad(t \in T) . \\
& |g(t)|<(\varepsilon / 3) 3=\varepsilon \quad(t \notin G) .
\end{aligned}
$$

The Lemma is now proved.

Proof of THe Rudin-Carleson TheOrem. $\varepsilon<\frac{1}{4}$ being fixed, denote by $\gamma(\varphi)$ any continuous function of analytic type associated to $\varphi$ by the Lemma. Starting with $\varphi_{0}=\varphi$ we put $\varphi_{m+1}=\varphi_{m}-\gamma\left(\varphi_{m}\right)$. We have

$$
\begin{aligned}
& \sup _{F}\left|\varphi_{m+1}\right|<\varepsilon \sup _{F}\left|\varphi_{m}\right|<\cdots<\varepsilon^{m+1} \sup _{F}\left|\varphi_{0}\right|, \\
& \underset{T}{\sup }\left|\gamma\left(\varphi_{m}\right)\right|<3 \sup _{F}\left|\varphi_{m}\right|<3 \varepsilon^{m} \sup _{F}\left|\varphi_{0}\right| .
\end{aligned}
$$


The series $\sum_{m=0}^{\infty} \gamma\left(\varphi_{m}\right)$ is therefore uniformally convergent on $T$; its sum $f$ is of analytic type and satisfies the relation $f(t)=\varphi(t)(t \in F)$. Moreover

$$
\sup _{T}|f(t)|<3(1-\varepsilon)^{-1} \sup \left|\varphi_{0}\right|<4 \sup _{F}|\varphi| .
$$

The theorem is now proved.

REMARK. The factor $M$ in the estimate (*) can easily be reduced to $1+\varepsilon$. In fact, given an open set $G \supset F$ and using (**) we can manage to have

$$
|f(t)|<\varepsilon \quad(t \notin G) .
$$

By the continuity of $f$, there is an open set $G^{\prime} \supset F$ such that $G^{\prime} \subset G$ and $|f(t)|<1+\varepsilon\left(t \in G^{\prime}\right)$. Thus we can have

$$
|f(t)| \geqslant 1+\varepsilon \quad \text { only if } t \in G \backslash G^{\prime} .
$$

Starting with $G^{\prime}$ we get $f^{\prime}$ coinciding with $\varphi$ on $F$, bounded by 4 where $\left|f^{\prime}(t)\right|>1$ $+\varepsilon$ only if $t \in G^{\prime} \backslash G^{\prime \prime}$ for an appropriate $G^{\prime \prime} \supset F$, with $G^{\prime \prime} \subset G^{\prime}$. Observing that the sets $G \backslash G^{\prime}, G^{\prime} \backslash G^{\prime \prime}, G^{\prime \prime} \backslash G^{\prime \prime \prime}, \ldots$ are disjoint and taking an arithmetic mean we get a function bounded everywhere by $1+2 \varepsilon$.

Proof of The First F. AND M. Riesz TheOrem. It is sufficient to prove that

$$
a_{0}=(2 \pi)^{-1} \int_{T} f(t) d t=0
$$

for, applying the same process to the function $e^{-i t} f(t)$, we deduce $a_{1}=0$, and next $a_{2}=0, \ldots$ and finally $f=0$. We shall follow the same pattern of proof as for the Rudin-Carleson Theorem.

Denote by $S$ the set $\{t \in T: f(t) \neq 0\}$. Given $\varepsilon>0$ let $e^{-A}=\varepsilon$ and let $h$ be a bounded real function equal to $-2 A$ on $S$ and such that $\hat{h}(0)=0$. There are such functions since $m\left(S^{*}\right)>0$. Let $p_{n}$ be the sequence of Fejér polynomials of $h$. We write as before

$$
p_{n}(t)=\sum_{k<0} \beta_{k} e^{i k t}+\sum_{k>0} \beta_{k} e^{i k t}=p_{n}^{-}(t)+p_{n}^{+}(t)
$$

where

$$
p_{n}^{+}(t)=\sum_{k>0} \beta_{k} e^{i k t}
$$

Then, boundedly,

$$
\operatorname{Re}\left(p_{n}^{+}(t)\right)=\frac{1}{2} p_{n}(t) \rightarrow \frac{1}{2} h(t)=-A \quad \text { a.e. on } S .
$$

Put now

$$
g_{n}(t)=f(t)\left[1-e^{p_{n}^{+}(t)}\right]
$$

The expansion of $g_{n}$ is of the form $\Sigma_{k>0} \gamma_{k} e^{i k t}$ and therefore $\int g_{n} d t=0$. Hence

$$
\begin{aligned}
\left|2 \pi a_{0}\right| & =\left|\int f\right|=\left|\int\left(f-g_{n}\right)\right|<\left|\int f e^{p_{n}^{+}}\right| \\
& <\int_{S}|f| e^{p_{n} / 2} \rightarrow e^{-A} \int|f|=\varepsilon\|f\|_{1} .
\end{aligned}
$$

Since $\varepsilon$ is arbitrary we have $a_{0}=0$ and the theorem is proved. 
Proof of the Second F. ANd M. Riesz Theorem. We may assume $a_{0}=0$. Let $F$ be a closed set of measure zero. Choose a decreasing sequence of open sets $G_{n} \supset F$ such that $\cap G_{n}=F$, and by the Lemma a sequence of functions $g_{n}$ of analytic type, such that

$$
\begin{gathered}
\left|1-g_{n}(t)\right|<1 / n, \quad t \in F, \\
\left|g_{n}\right|<3 ; \quad\left|g_{n}(t)\right|<1 / n \quad \text { for } t \notin G_{n} .
\end{gathered}
$$

Then, boundedly, $g_{n} \rightarrow \chi_{F}$ (characteristic function of $F$ ). Hence $0=\int g_{n} d \mu \rightarrow$ $\int \chi_{F} d \mu=\mu(F)$. This proves that $\mu$ is absolutely continuous.

The author would like to thank Y. Katznelson for a useful conversation.

\section{REFERENCES}

1. L. Carleson, Representations of continuous functions, Math. Z. 66 (1957), 447-451.

2. R. G. Douglas, Banach algebra techniques in operator theory, Academic Press, New York, 1972.

3. P. Duren, Theory of $H^{p}$-spaces, Academic Press, New York, 1970.

4. K. Hoffman, Banach spaces of analytic functions, Prentice-Hall, Englewood Cliffs, New Jersey, 1962.

5. Y. Katznelson, An introduction to harmonic analysis, Dover, New York, 1976.

6. B. Oksendal, A short proof of the F. and M. Riesz Theorem, Proc. Amer. Math. Soc. 30 (1971), 204.

7. F. Riesz and M. Riesz, Über die Randwerte einer analytischen Funktion, $4 \mathrm{e}$ Congrès des Mathématiciens Scandinaves (Stockholm, 1916), pp. 27-44.

8. W. Rudin, Boundary values of continuous analytic functions, Proc. Amer. Math. Soc. 7 (1956), 808-811.

9. Real and complex analysis, McGraw-Hill, New York, 1974.

Department of Mathematics, State University of New York, Stony Brook, New York 11794 